\title{
THE FAUCIAL TONSILS AND ACCESSORY SINUSES OF THE NOSE IN RELATION TO FOCAL SEPSIS.
}

\author{
$B y$ F. C. W. CAPPS, F.R.C.S. \\ (Surgeon with charge of Out Patients, Throat Department, St. Bartholomew's Hospital, \\ London, etc.)
}

The indications for the removal of tonsils and adenoids are subjected in these days to the light of much fiercer criticism, lay and medical, than was the case some twenty years ago, and no section of the subject more fiercely perhaps than that relating to focal sepsis. The scope of this paper is limited to a discussion of this section and at the outset it is wise to be quite clear what we mean by the term.

An excellent definition is given by Christian in Osler's text-book of Medicine (I938), namely "A term used to express the idea of a local chronic infection that is causative of distant and important manifestations."

He concludes "Tonsils and teeth more often than any other source serve as focal infections but chronic infection 'localised' anywhere in the body may have this relationship." That "teeth, tonsils and tummy," to put the three chief offenders in alliterative form, can all fulfil such a rôle is established by numberless well authenticated investigations, case series and individual records but it is equally true that many operations for removal of such foci have been performed with too little consideration of the pros and cons or as a pure speculation.

It is the aim of this paper to emphasise those conditions in which sepsis in the upper respiratory tract appears to be a true causal factor and to establish the criteria for surgical or other interference.

Christian goes on to say, "The earlier belief in the great frequency and importance of focal infection as an explanation of many acute and chronic diseases now is on the wane. Many are sceptical of this relationship except in relatively few cases." Yet the frequency with which many departments of all hospitals still refer cases to the ear, nose and throat surgeon for an opinion as to the presence or absence of such a focus, and in cases thought to be positive, request his intervention, leads one to suspect that success or improvement must be sufficiently common to justify the conception of "focal sepsis."

Such references come chiefly, of course, from the physicians and usually in cases of affections of the joints and fibrous tissues, the kidneys, thyrotoxæmia, and occasionally diabetes, but they are also quite numerous from the eye department, the skin department, the children's department and the orthopædic department. Much work has been done also on sepsis, particularly in the paranasal sinuses, in relation to mental disease.

Horder, writing in the Oxford Text-book of Medicine, I937, says, "In focal sepsis there may be few or no symptoms referable to the local site of infection. Yet the resulting toxæmia may manifest itself as fibrositis, arthritis, anæmia, rarefaction of bone, loss of weight, pyrexia or a combination of these. In focal sepsis pyrexia is not a marked feature except in some cases of coliform infection of the urinary tract and in a minority of dental apical infections. The likely sites are nose and nasal sinuses, nasopharynx, teeth, tonsils, etc. ; chronic infection may lurk in certain of these situations, even in the absence of gross macroscopic lesions. The tonsils, for 
example, when not enlarged may contain pus or inspissated secretion in their crypts. A scarred and ragged tonsil or one which has venules on its surface is always suspect, especially when a lymph node is palpable."

This is a very fair statement but it might be amplified to the extent that in nearly every genuine case a very careful search of the history, especially in the past, will almost always elucidate one or multiple indications of the site of infection and may suggest the use of such collateral aids in localisation as X-rays and bacteriological examination. In the regions under consideration here the last named may be made on swabs, expressed material, tonsil puncture or aspiration material from a suspected sinus. The blood picture and sedimentation rate may also help to indicate an infective cause of disease.

It cannot, however, be too strongly stressed that when all possible useful investigations have been made the final and only adjudicator must be the physician in charge. On him rests the onus of deciding:-

I. That the disease has its origin in a septic focus.

2. Of weighing the relative merits of alternative foci.

As ear, nose and throat surgeons we can advise but must not be dogmatic.

With regard to bacteriology the streptococci are the most numerous group of organisms found. Although it is stated that hæmolytic streptococci are present in a high proportion of throats it is probable that they are only present in any number in acute phases. The work of D. and R. Thomson (I929) suggests that the flora of the throat are constantly changing in the same individual. It is probable that as more accurate serological classification of hæmolytic streptococci is attained certain strains may be found to predominate in certain conditions. Pneumococci are also a frequent inhabitant of the tonsils. The tendency of modern bacteriology is to believe in the temporary occurrence of a bacteræmia rather than a persistent toxæmia and such a consideration may greatly influence us in the future in timing our surgical procedures. This will be referred to in greater detail later.

D. and R. Thomson, after a very comprehensive survey of the history of the bacteriology of the tonsils and numerous investigations of their own, concluded that " the tonsils are of little value, they are frequently septic foci and a constant source of systemic infection"'. Against this must be placed the view of many experienced laryngologists that the tonsils are but part of the primary lymph drainage of the upper respiratory tract and that it is as irrational to remove them as to dissect out enlarged cervical glands when infected. Particularly in children, it is argued, tonsils become enlarged as a result of unhygienic surroundings nasal infection and bad nutrition and if these are corrected the tonsils return to a normal appearance and bacteriology. The poor condition of the children is due to these conditions and the tonsils are only one of the manifestations. It is certainly unjustifiable to condemn a child to enucleation of the tonsils without first proving that change of environment and better food will not correct the general picture and with it the appearance of the tonsils. Many of us also must be familiar with the improvement in the appearance of the throat when a chronic nasal infection or obstruction is eliminated. For the evidence of the benefit derived by children from tonsillectomy reference should be made to the analysis of 500 cases at Great Ormond Street, by Patterson and Bray (r928). These authors stress the importance of including the lingual tonsil in the tissues removed. The report of Albert D. Kaiser (I932) on the observation of 5,000 children, half of them tonsillectomised, over a period of ro years is also in favour of the tonsillectomised where the incidence of rheumatism is concerned. 
A prominent argument against "focal sepsis" is the disparity between the frequency of foci and resulting disease. On the other hand this may be accounted for by the natural resistance and immunity of the patient under ordinary conditions. It is probable that some abnormal circumstance must supervene to upset the balance. The author can speak from personal experience of attacks of fibrositis of neck, shoulder and sacro-iliac region which came on after some strain or twist (often quite trivial) in these regions and which he afterwards realised had closely followed faucial inflammation in the days before he was tonsillectomised. Since then the occurrence has been less frequent and has always cleared up as if by magic when an intercurrent and often unrealised infection of an antrum or ethmoid evacuated itself a few days after the onset.

Thus injury, chill or some intercurrent and debilitating illness may give the hidden focus its chance.

We must now discuss the investigation of a suspected case and deal with the various groups in greater detail.

The author can well remember the reply of one of his teachers, the late Frank Rose, who, when asked what he considered a "septic" tonsil, replied "I don't know". There is no recognised definition of such an entity, bacteriology has not so far helped us to differentiate between the harmless and the potentially dangerous tonsil, size is no criterion. The expression or aspiration of frank, creamy pus (as opposed to the almost inevitable cheesy débris) is a more certain indication of evil.

First in importance comes the history of the case. Has there been a bad, single or recurrent attack of sore throat or tonsillitis? Have there been one or more attacks of quinsy and if so, on which side or sides? Has there been a previous operation for removal of tonsils and adenoids? This is of prime importance if on examination such operation is found to have been incomplete. Rhoads and Dick of Chicago (I928) investigated the danger of tonsil stumps. In a double series of cases they made bacterial counts, microscopic examination, and tabulated the systemic relations of tonsils removed for the first time and removed stumps. In the first series the bacterial count per gramme of removed tonsil averaged 5,693,000 as compared with 7,341,000 in the stumps, or remnants. In the latter the outstanding microscopic change was fibrosis and many showed the crypt orifices closed and the crypts themselves dilated by exudate and organisms. Under the heading of imperfect operations must be included the use of caustics such as London Paste and diathermic coagulation. Both methods are nearly always incomplete and there is the added danger that the mucous membrane tends in these cases to grow in and cover the remnant so that it is no longer evident macroscopically except in attacks of inflammation or to the experienced eye of a suspicious clinician. It is probable that a far higher proportion of the "failures" after removal of tonsils and adenoids as a focus of sepsis are due to incomplete operations and relatively few to the well recognised tendency of some cases to increase the remaining islands of lymphoid tissues in Waldeyer's ring after enucleation of the faucial tonsils and adenoids.

In the history also should be included any reference by the patient to what is often described as "gushes" of foul taste or smell at the back of the tongue or in the oro- and nasopharynx. Patients may have noticed that the lymph nodes behind and below the angles of the jaw are subject to recurrent enlargement although there may have been no very obvious "flare-up" in the throat. 
Examination should start by careful inspection. Swellings can sometimes be seen in the neck, a general impression of teeth and tongue can always be obtained without instrumental aid and many a patient will display the throat. If they will, a careful note should be made of any general blush or localised red patches on the anterior pillars of the fauces. These are of great significance in suggesting latent infection in the underlying tonsil. They are not of such significance after a spatula has been pressed on the back of the tongue or fauces or the patient has "gagged". Any pillar is likely to look red after these manœuvres.

Before pressing on the pillars or retracting them and thus rendering the throat irritable it is wise to do a posterior rhinoscopy to exclude adenoids or pus coming from the back of the nose, and a laryngoscopy which should include a careful inspection of the lingual poles of the faucial tonsils and the lingual tonsils themselves.

In cases of focal sepsis and even in the ordinary recurring sore throat it is more often the small buried and hidden tonsil which is to blame. The best way to reveal this and study its secretion is to use two Lack's spatulæ, choosing a size to suit the patient. With one the back of the tongue is depressed and the second is used to press back the anterior pillar and evert and press on the tonsil. This may produce concretion, probably mostly epithelial debris and therefore harmless, foul smelling material or frank pus. The appearance of the tonsil itself is often suspicious. If whole and untouched by previous operation the most suspicious variety is the rather pale pink, soft looking tonsil with the mouth of the crypts poorly defined and the surface sometimes studded with exudate at the site of these crypts. The scarred tonsil remnant must always be suspect for the reasons given above.

Finally, make a careful rhinoscopy with deliberate inspection of supreme, middle and inferior meati and compare the relative translucency of the frontal sinuses and maxillary antra. The presence of pus or polypi in the nose is conclusive evidence of sinus trouble, though with polypi allergic tendencies may account for their formation. Relative opacity is a helpful but far from certain sign. It is of more value in the antra, which are usually present on both sides and equal in size. (It is probably needless to point out that the upper denture must be removed first). But darkness, and even X-ray opacity, unless it shows a definite fluid level, still leaves you uncertain of the contents, and the only way to be sure in the antra is to perform a proof puncture. The straight cannula is the best and it is an advantage to have the nozzle end of a size to fit a standard Record syringe. This enables aspiration of the contents by a 5 or ro c.c. syringe. It is easily performed, even in children, under local anæsthesia.

In the author's opinion the sinuses usually give rise more to local trouble and headache, but now and again they are certainly responsible for distant manifestations especially fibrositis. Carl M. Añderson (I930) reviewed 400 cases. He found that a suppurating sinus was not a significant factor in focal infection. He suggests that anatomical and pathological reasons explain this, e.g. the natural drainage is good, the lining mucous membrane is ciliated, contains few glands and is thinner than elsewhere in the nose: The secretions in the nose and accessory sinuses resist bacteria and toxins soluble in water, and finally, the lymphatic nodes and vessels are relatively few. In 200 cases of sinusitis without other foci of infection there were only three cases of rheumatism and one of bronchitis, which were coincident with acute infection of the upper respiratory tract. In a second group of 200 cases who had foci in one or more regions besides the nasal sinuses 26 per cent. presented complications which may have been caused by 
focal infection. In his experience teeth, tonsils, prostate gland and other foci are considered of more importance than the sinuses.

Some interesting work has been done by Gording and Björn Hanson on the question of the leucocytic blood picture and sedimentation rate in cases of rheumatism. There seems evidence to suggest that where foci of infection are present this may be indicated and in a number of cases after removal of the focus. tonsillar or dental, the blood picture improved.

Perhaps the most successful group in which the tonsils seem to play the part of focal infector are the cases of nephritis. A. C. Alport (I932) believes the percentage of tonsillitis in cases of nephritis to be as high as 85 per cent. with the streptococcus as the usual organism. Mygind in a paper on the relation of the tonsils to acute rheumatism and hæmorrhagic nephritis found the results more convincing in the latter condition. There should be a clinical history of tonsillitis and a history of quinsy is still more significant. Characteristically a second or subsequent attack immediately precedes the nephritis. The most favourable cases are those in which the nephritis is the first acute attack and not an exacerbation of a chronic condition. The simpler the clinical expression of the nephritis, e.g. hæmaturia, cylinduria, and minimal albuminuria, the better the chance of cure by removal of the tonsils. Hyperpiesis, a lot of albumen and œedema are unfavourable factors, while in the nephrotics, with massive albuminuria and œdema without hæmaturia the prospect of success by removal of a septic focus is even less. Alport however considers that most success is to be expected in subacute nephritis with much œdema.

The cases should be watched and treated medically and the tonsils removed as soon as they are well or when, after prolonged medical treatment, improvement becomes stationary.

These cases are very prone to bleed, more than the ordinary tonsillectomy, and particular attention should be paid to every small bleeding point. When there is a general ooze which cannot be stopped it is best to stitch the pillars together over a pack, preferably of "bipped" gauze which can be left for several days without becoming foul.

As it has recently been demonstrated by S. D. Elliott (I939) that operation on a septic focus may give rise to bacteræmia the advisability is suggested of freeing the area of operation from active infection as much as possible by antiseptic sprays and mouthwashes and if possible choosing a moment when culture shows the concentration of hæmolytic streptococci, or other predominant organism to be low. Elliott's first work concerned the removal of septic teeth but he has also reported a series of Ioo tonsillectomies, both by dissection and guillotine, in which a blood culture was performed immediately before and after the operation. In nearly 40 per cent. the post operative culture showed a bacteræmia, the causative organism being very varied. There is no reason why a precautionary course of sulphanilamide where hæmolytic streptocci are concerned, or M. \& B. 693 in pneumococcal infection, should not be given two or three days before operation. A single preoperative dose is of no value and concentration must be carefully checked in continued doses.

In the rheumatic group the importance must again be stressed of a clinical history pointing to tonsils or sinuses. Owing to their often obscure manifestations and the fact that such a thing as a bad cold or attack of "influenza" may easily be forgotten; it is not always so easy to get a history in the latter case. In 
clinical examination the maxillary antrum is the only cavity which can be explored with any ease, and good stereoscopic X-rays may be the only guide to an ethmoid, frontal sinus or sphenoid, although there is usually some change to be noted in the nose or postnasal space such as a swollen turbinal bone, polypoid mucous membrane or a trickle of pus.

The results in "rheumatism" show great variation owing to the great diversity of conditions which become grouped under this heading. In fibrositis or muscular rheumatism the effect of removal of a septic focus in tonsils or sinuses shows a high percentage of success, especially in the neck, shoulders and back. In the acute rheumatism of children it is well known that removal of the tonsils after the initial attack will not prevent recurrent attacks but when the initial attack was preceded by an acute tonsillitis and it is known that subsequent attacks are also likely to be so preceded surely it is better to remove this focus. Although it is also known that tonsillectomy does not protect against a primary attack of acute rheumatism Kaiser's series showed a 30 per cent. lower incidence in the tonsillectomised. Nabarro and Macdonald (I929) studied the relation of the bacteriology of the tonsils in relation to rheumatism in children. They found that there was no material difference between the bacteriology of the rheumatic and the nonrheumatic and came to the conclusion that this absence of difference agrees with the theory that there is no specific streptococcus which is the cause of rheumatism, but that the condition is due to a hypersensitiveness resulting from repeated small doses of toxin. Inasmuch as the tonsil is probably a common source of such toxin its removal may be a valuable prophylactic and therapeutic measure in spite of the lack of difference in bacteriology. Reidar Gording concluded that rheumatism was tonsillagenous mainly under the age of 35 .

In cases of arthritis where there is a history pointing to tonsil or sinus much depends on the degree of anatomical change which has taken place. In the early cases with only one or few joints involved the results may be excellent. In more advanced cases and the rheumatoid cases cure cannot be expected but progress is not infrequently arrested and pain relieved.

Where there is a clear indication of relationship with the tonsils or frank pus is found in a sinus it would seem that action must always be indicated. Where therc is reasonable doubt in the case of the tonsils, but no more obvious focus can be found, it is probable that the interests of the patient are best served by an operation which, although somewhat speculative. is reasonably free of danger and may save severe and crippling deformity if performed in time.

The time for operative procedures should be a quiescent and afebrile period. In acute rheumatism the cardiac condition must necessarily be a controlling factor, especially in regard to the anæsthetic. Incompletely compensated cases must be got into as efficient a state as possible before operation is considered.

In cases of toxic goitre there is a not infrequent history of recurrent tonsillitis and in others, although the history may not be so certain the appearance of the tonsils is certainly far from innocuous. Their removal when done at the proper time and with due safeguards, under the control of a physician, is almost always beneficial. It is not the business of the throat surgeon to say whether such operation should precede or follow a thyroidectomy if such is to be performed. Fraser (I934) says of treatment "infected teeth, sinuses and tonsils likewise must be treated. The tonsils are often large and red in toxic goitre and subside as recovery from toxicity takes place, but when the tonsils contain pus tonsillectomy should 
be performed. In milder cases this can be done with advantage before thyroidectomy but in the more severe cases it may be wiser to wait until after the thyroid condition has improved as a result of thyroidectomy for the operation of tonsillectomy seems to be nearly as severe a strain as the thyroidectomy itself."

It is certain that modern pre-anæsthetic medication has considerably reduced the severity of operations in these cases. Avertin 0.I grm. to kilo of body weight with or without morphia is ideal, and many cases can then be done without any ether at all, although it is better to give the latter rather than have the patient coughing and straining. Chloroform must never be given. Congestion and cyanosis must be avoided and the pulse rate carefully watched for sudden rise in rate or fibrillation. The patient need know nothing of the operating theatre or its approaches. The operation should be done as expeditiously as possible and this postulates good facilities. Dissection should always be done, as control of bleeding is of great importance. If endotracheal anæsthesia is not available a suction apparatus should be available for the constant protection of the airway. The operation should always be preceded by rest and medical treatment and while improvement is being maintained no operation should be contemplated. They are the last cases to be put into hospital or nursing home the night before the operation.

Diabetics leading an insulin life now and again become unbalanced as a result of septic infection and if the number of units of insulin required does not return to the former level it may be necessary to remove or clear the focus of infection. The important factor in these cases is the immediate pre-operative treatment and punctuality of operation to coincide with this. (50-70 grns. of glucose, followed in I5 minutes by $20-50$ units of insulin, is said by Hewer to reduce the risk of diabetics almost to that of normal cases).

In diseases of the eye the conditions where sepsis in the upper respiratory tract seems to bear a direct relationship are keratitis profunda, some cases of uveitis, central retinal hæmorrhage and exudate. Authorities differ in ascribing sepsis in the sphenoid or a posterior ethmoid as a cause of retro-bulbar optic neuritis.

The attitude of the physicians in the skin department appears to be that many skin affections are influenced by the general health of the patient and inasmuch as removal of a septic focus may improve the general health, so the skin condition may benefit. The disappearance of pustular acne from the region of the mouth and face after the removal of a septic pair of tonsils must be an experience common to many throat surgeons.

A large number of cases of "painful feet" are referred by the orthopædic department and the author recently had the satisfaction of hearing that the eradication of longstanding sinus infection in such a case had enabled a youth, formerly almost crippled for several years, to join the regular army and be well and fit after a year's service.

The pioneer work in the establishment of the relationship between focal sepsis and the "septic psychoses" was performed by William Hunter (I927) in this country and Dr. Cotton of New Jersey (U.S.A.) and there has been recent valuable work by Drs. Graves and Pickworth of the Hollymoor Asylum (Birmingham). They found that sinusitis was found on admission and at autopsy in cases associated with many types of mental disorder, and demonstrated improvement in certain cases following treatment of the sinusitis. 
On the other hand Jowett (I936) in an examination of 500 routine cases of mental disease proved nasal sinus infection in 7.6 per cent. This compared with an incidence of not less than 5 per cent. in a control series of 184 mentally normal individuals over the same three year period. The writer considered that before conclusions can be drawn from post-mortem pathological material collected in mental hospitals the great incidence of sinus infection at some period of life in all individuals must be considered. No reliable information about the residua of past infection in the sinus of people dying outside mental hospitals is at present available. Further, in the tabulation of specimens showing sinus disease in mental cases the effect which the disease causing death might have upon the conditions of the accessory sinuses must be carefully considered.

It would appear that a good deal more work will have to be done on this question before a balanced opinion can be expressed.

To summarise:-

There seems sufficient evidence to justify the conception of the tonsils and accessory sinuses of the nose amongst others as sources of focal infection.

The most convincing results are obtained in nephritis and some cases of muscular rheumatism and arthritis. Good results are also obtained in cases of toxic goitre. Results in other conditions are occasional only and more uncertain.

It is important to elucidate a history of previous trouble in these organs followed by an attack more definitely associated with the onset of the disease in which they are suspected of complicity.

Whenever possible, operation should be deferred to an afebrile or quiescent period or at least until improvement is at a standstill.

In certain conditions, nephritis and thyrotoxæmia, more than usual bleeding may be expected at operation. A good anæsthetic is essential with adequate airway, plenty of oxygen and just as much anæsthetic as is necessary and no more.

If operation is indicated it should be a complete removal in the case of the tonsils and adequate drainage in the case of an accessory sinus. Complete removal of the faucial tonsil is best performed surgically. Cautery and electrocoagulation are unsatisfactory and local therapy by pressure and suction are valueless.

Lavage of a sinus, such as the antrum, may be tried before operative drainage.

REFERENCES.

ALPORT, A. C. (1932), The Lancet, 1, 1247.

ANDERSON, CARL M. (1930), Jour. Amer. Med. Assoc., 94, 24.

(1939), Proc R S.M. Ar

FRASER, FRANCIS R. (1934), a series of lectures on Toxic Goitre delivered before the Derby Medical Society during May, 1934, GORDING, REIDAR, R. Fraser and Sir Thomas P. Dunhill, S

GORDING, REIDAR, Acta Oto-laryngologica, Vol. vi, fasc 3-4. Supplement xvii.

GRAVES, T. C. and PICKWORTH, F. A., “ Sinusitis in the Etiology of Mental Disorder," Jour. Lar. and Otol., 43, 545.

HEWER, C. LANGTON, "Recent Advances in Anæsthesia and Analgesia."

HUNTER, WILI.IAM (1927), "Relation of Focal Sepsis to Mental Disease," Brit. Med. Jour. (Sept.).

JOWETT, RONALD E. (1936), " "Sinus Sepsis and Mental Disorder," Jour. of Men. Sci. (Jan.).

KAISER, ALBERT D. (1932), “ Children's Tonsils-In or Out," J.'B. Lippincott, London.

MYGIND, S. H. Acta Oto-laryngologica, Vol vi, fasc 4 .

NABARRO, DÄVID and MACDONALD, R. A. (1929), Brit. Med. Jour. (Oct.).

PATTERSON, DONALD and BRAY, J. W. (1928), ". Tonsillar Hypertrophy and Infection as a Factor of Ill-Health," The Lancet, 2, 1074 .

RHOADS, PAUL S. and DICK, GEO. F. (1928), Jour. Amer. Med. Assoc., $91,16$.

THOMSON, D. and THOMSON R. (1929), Annals of the Pickett-Thomson Lahoratory, 5. 